\title{
nature
}

4 December 1997 Volume 390 Issue no 6659

\section{A time for responsibility}

Researchers have good reasons to be nervous of proposals that all life science research grant applications to Brussels be scrutinized for their potential implications. But careful handling could produce benefits for all.

T here is, at times, something almost Kafkaesque about the way in which the scientific community has recently found itself in the dock, accused of vaguely worded crimes, unable fully to comprehend the nature of the charges it faces, yet threatened with dire sanctions if found guilty by public acclaim. Such, at least, tends to be the feeling generated in researchers who find themselves bemused by the growing desire of both governments and elected politicians to embrace the discipline of bioethics. It is certainly the reaction already provoked in some by the news that the European Commission is likely to require all grant applications in the life sciences to be assessed for their moral acceptability (see page 433).

The dangers in such moves are clear. The most obvious lie in the extra burdens imposed by any new bureaucratic requirement. But requiring all applications to pass an ethics test could in principle impose a stifling moral and intellectual orthodoxy on research, prejudging what can and cannot be studied in potentially dangerous ways. Anyone claiming that this is an exaggeration needs only to look at the powerful influence of the anti-abortion lobby on critical areas of biomedical research in the United States.

But those increasingly tempted to reach for a gun at the mention of the word bioethics should pause before doing so. It may be unrealistic to try to imbue the average laboratory scientist with the foresight needed to predict the full social impact of his or her discoveries, let alone their moral and ethical implications. But this does not absolve the researcher of all responsibility for thinking about the potential impact of those discoveries. It merely suggests that the link between discovery and application is a complex process in which both personal and collective judgements have important roles to play.

In this context, the important question to raise about the new steps being proposed to the European Commission is not whether they are either desirable or necessary - the global response to the reports of the cloning of Dolly the sheep should be sufficient to dispel any doubts on both scores - but how they can be made to work most effectively. Here some important guidelines need to be followed.

First, it is essential that ethical assessments are based on sound science. One implication is that the scientific community has a clear responsibility to monitor the work of ethics committees at all levels, aware of the danger that, in highly charged debates, there can be a tendency for bad science to drive out good, sometimes exaggerating dangers for inappropriate reasons.

Second, the process must be sufficiently transparent to inspire the confidence of both the public and the research community. There is a need to overcome the culture of secrecy that, while perhaps protecting political reputations, can also undermine sound decision-making by limiting the scope of public scrutiny.

Third, there must be clear mechanisms to distinguish questions of ethics from those of safety. Although there are clear areas of overlap, there are also significant dangers of confusing the two in deciding how they should be handled.

Finally, an active effort must be made to eschew an excessively formulistic approach to ethical questions, remembering that one man's set of clear principles can rapidly become another man's moral and intellectual strait-jacket. A pragmatic approach has its own dangers; but these are usually easier to rectify than one that is cast in stone.

If these principles are ignored, there is a danger that what started as a well-intentioned effort to bridge the gap between the need to respect scientific freedom and legitimate public concern about the impact of some new scientific discoveries will have backfired. Excessive bureaucratic requirements that produce few concrete results will merely become one more impediment to Europe's scientific creativity.

But if these principles are followed, Europe will have demonstrated its ability to develop a sound approach to a key aspect of science policy appropriate to the political and social demands of the new millennium. Both science and the community that supports it will be better off.

\section{Preprints and Nature}

\section{Exposure of preprints on servers does not preempt their submission to this journal.}

$\mathrm{n}$ some scientific disciplines it is common for draft papers to be circulated to colleagues before publication - a practice that has become formalized with the advent of electronic preprint servers pioneered at the Los Alamos National Laboratory. Superstring theorists and others working at high energies were in the vanguard, and it may be relevant to note that these communities are, within the spectrum of disciplines, unusual in their approach to peer review — for example, many high-energy physics experimental papers are refereed extensively within the large teams of collaborating authors before being circulated.

In other areas, where the communities are larger and the variability in quality and sheer volume of preprinted material somewhat daunting, preprint servers are active but appear to be considered less useful for those reasons. And in some areas of research, life is too fraught with competition for preprints to be advisable. Nevertheless, preprint servers are, for some, part of the means of routine scientific discourse. They seem unlikely to displace the need for the selection and broader propagation of results provided by most journals.

There is no conflict between preprint circulation and submission to Nature - we only request that submitting authors inform us where and when a preprint has been placed on a server. But we do actively discourage prior exposure of results in the public media, given our conviction that the breadth and quality of coverage is strengthened by our embargo policy (for a statement of embargo policy and sanctions, see "author information" at www.nature.com). But Nature is more than just an archive. So we reserve the right not to publish papers whose results have already been disseminated and discussed beyond the ranks of specialists. And, as with conferences, our policy is that scientists wishing to publish in Nature are required not to encourage or cooperate with media coverage before publication, while communicating freely with other researchers in whatever way seems appropriate. 